\title{
La pervivencia del Musteriense en la mitad sur peninsular durante el Pleniglacial Superior. Una revisión crítica de las dataciones
}

\author{
almudena Villar Calvo*
}

RESUMEN

El presente artículo cuestiona la pervivencia de los Neandertales y de su tecnología Musteriense en la mitad sur de la Península lbérica durante el

Pleniglacial Superior wurmiense, criticando buena parte de las fechas obtenidas en los principales yacimientos de la región, ya sea por los métodos por los que se han obtenido o bien por la procedencia y la naturaleza de las muestras.

\section{PALABRAS CLAVE} Paleolítico, Musteriense, Neandertales, Dataciones, España, Portugal.

\section{ABSTRACT}

This paper calls into question the survival of Neanderthals and their Mousterian technology in Southern Iberia in the Upper Pleniglacial, carrying out a critical review of the dates of mousterian levels in the main sites of this area, whether by dating methods or by the origin and the nature of sample.

\section{KEY WORDS}

Palaeolithic, Mousterian, Neanderthals, Dating, Spain, Portugal.

La idea de una transición "retardada" del Paleolítico Medio al Superior en la mitad meridional de la Península lbérica esta firmemente asentada en la comunidad científica internacional, lo que conlleva la creencia en una larga pervivencia del Musteriense hasta pasado el 30.000 B.P. y en la existencia de una población relicta neandertal en este área, que implica la coexistencia durante un largo período de tiempo con las poblaciones modernas del resto

\footnotetext{
* Colaboradora del Departamento de Prehistoria e Historia Antigua de la UNED.
} 
de Europa. La generalizada aceptación de esta hipótesis ha llevado a

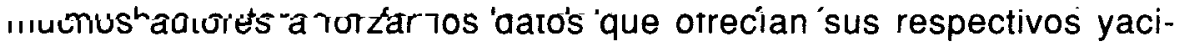
mientos para acomodarlos a la corriente de investigación, se han trazado fronteras ficticias entre el territorio Auriñaciense y el Musteriense y, casi siempre, las explicaciones a esta perduración han sido endebles cuando no inexistentes.

El planteamiento de este modelo de pervivencia se ha basado, fundamentalmente, en una serie de fechas obtenidas con diferentes métodos cronométricos y en la idea obsoleta de la inexistencia o extrañeza de industrias asimilables a un Paleoítico Superior inicial. En este artículo pretendemos revisar críticamente las dataciones que se esgrimen como pruebas de la prolongada supervivencia del Musteriense en el Sur de la Peninsula y, sin intentar ser exhaustivos, si queremos dejar constancia de la debilidad de los cimientos sobre los que erigimos nuestras teorías.

Analizaremos en primer lugar el yacimiento "culpable» en gran parte del triunfo de este modelo, la cueva de la Carihuela (Granada), que posee una impresionante secuencia que abarca desde finales del Pleistoceno Medio hasta la Edad del Bronce (Vega, 1988; Vega y Carrión, 1993; Vega et al.,1988; Vega et al. 1997), que sirve de referencia al resto de yacimientos de la zona (Vega, 1993) y que proporcionó durante la investigación de la Washington State University una serie de fechas TL sobre sílex quemado y otros restos (Fremlin, 1974; Göksu et al., 1974), la problemática de estas dataciones ha sido tratada en extensión en la Tesis Doctoral del actual investigador del yacimiento (Vega, 1988) y que se puede resumir en dos puntos: por una parte, la falta de relación con niveles estratigráficos precisos de las muestras tomadas y por otra, la experimentalidad del método en estos años setenta que conllevaba un error de rejuvenecimiento de las fechas obtenidas y que supuso que de las 80 muestras tratadas sólo hayamos conocido 26 dataciones, seguramente por la indefinición de las restantes. Así pues, esta serie sólo puede considerarse orientativa, dando un intervalo aproximado entre el 90.000 y el 13.000 B.P. para las unidades XII a IV. Aunque en la actualidad se han obtenido diversas dataciones radiométricas, para el nivel III. $1=12.320 \pm 60 \mathrm{BP}$ (Beta74389 AMS), para el nivel VI.9 $=45.200 \pm 1.270 \mathrm{BP}$ (Beta-74381 AMS) y para la base de la secuencia en el Área Exterior nivel D0 $=117.000 \pm$ $41.000 \mathrm{BP}\left({ }^{230} \mathrm{Th} /{ }^{234} \mathrm{U}\right)$ y $146.000 \pm 1.700 \mathrm{BP}$ (U-series) (Vega et al. 1997), no contamos por el momento con ninguna fecha para las Unidades $V$ y IVb, las últimas con niveles Musterienses y cuya formación es compleja, teniendo en cuenta, además, que aún no conocemos la duración de la fase erosiva que separa las unidades $\mathrm{VI}$ y $\mathrm{V}$. 
Por lo que respecta al yacimiento del Boquete de Zafarraya (Málaga),

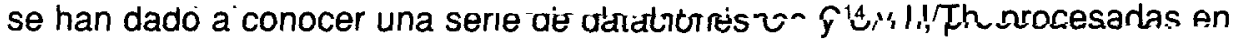
el Laboratorio de Gif-sur-Yvette (Hublin J.-J. et al., 1995), los niveles artificiales I ( 3 a 7 ) han proporcionado tres fechas U/Th, 25.100 $\pm 1.300 \mathrm{BP}$, $26.900 \pm 2.700 \mathrm{BP}$ y $28.900 \pm 3.600 \mathrm{BP}$ y una fecha $\mathrm{C}^{14} 29.800 \pm 600 \mathrm{BP}$; el nivel I (8) ha dado una fecha U/Th de $31.700 \pm 3.600 \mathrm{BP}$ y $\mathrm{C}^{14}$ de $31.800 \pm 550 \mathrm{BP}$, y por último el nivel $\mathrm{D}$ que ha aportado una fecha U/Th de $33.400 \pm 2000 \mathrm{BP}$. Aquí sólo queremos interrogarnos sobre la industria típica del Paleolítico Superior que aparece en el yacimiento como las dos laminillas retocadas encontradas a unos $10 \mathrm{~cm}$. a techo de los niveles Musterienses, cuya presencia ha sido explicada como intrusiones provenientes del nivel revuelto superior (Hublin et al., 1995: 934), aunque el primer nivel ha sido descrito como muy compacto (Medina et al., 1986: 96), si estas piezas son consideradas intrusivas, quizá también pudieran serlo los dientes sobre los que se han realizado las series de Uranio; también nos gustaría saber más sobre el alargamiento en los soportes, sobre la punta de Chatelperron y la lámina del nivel $D$ y sobre la colección de piezas óseas clasificadas como punzones. Creemos que sería necesaría la publicación de un estudio estratigráfico pormenorizado antes de poder admitir la credibilidad de estas dataciones. Además la utilización de biomarcadores que apoyen estas fechas tan recientes ya han sido contestados en otra parte (Cortés et al., 1996), como la ausencia de Allocricetus bursae, especie que no parece ser el mejor argumento bioestratigráfico.

Otro yacimiento malagueño, Cueva Bajondillo, ha venido a sumarse en los últimos años a los sitios que proponen fechas muy recientes para el Musteriense andaluz (Cortés y Simón, 1997). Los niveles arqueológicos se dasarrollan sobre los travertinos mediando una superficie de erosión química; la formación travertínica de Torremolinos ha sido datada entre 25.300 y $26.500 \mathrm{BP}$ (ESR) y en $27.300 \pm 1.700 \mathrm{BP}$ (U-series), lo que supondría fechas aún más recientes para los seis niveles Musterienses, como aún no conocemos los datos faunísticos, polínicos o sedimentarios o dataciones realizadas directamente en el yacimiento que puedan corroborar esta cronología, creemos que deben ser tomadas con precaución. Esperamos que las investigaciones de Cueva Bajondillo sigan aportando datos, sobre todo, por la presencia de un nivel Auriñaciense s.l. que nos ayudará a comprender la realidad de lo que ocurrió en Andalucía en la transición al Paleolítico Superior.

Los yacimientos gibraltareños no parecen arrojar mucha luz al problema y únicamente contamos con el ensayo de correspondencia con la secuencia de Carihuela que realizó Vega Toscano (Vega, 1990 y 1993) que relacionaba el nivel $V$ de Carihuela, ya en el Pleniglacial Superior con el 
último nivel musteriense de Gorham's Cave — nivel G-y con el nivel 1 de Devil's Tower. Las dataciones radiocárbonicas obtenidas en Gorham para el nivel musteriense $\mathrm{G}=47.700 \pm 1.500 \mathrm{BP}$ y $49.200 \pm 3.200 \mathrm{BP}$ y para el nivel D del Paleolítico Superior s.l. en torno al 28.000 BP, no casan con la secuencia andaluza y son escasamente tenidas en cuenta. Esperamos que los nuevos trabajos realizados en la zona ayuden a solucionar el problema planteado (Díaz, 1994; Hoyos et al., 1994).

En cuanto a los yacimientos de la Comunidad Valenciana, nos encontramos con Cova Negra (Valencia), en la que se han distinguido seis fases sedimentoclimáticas con catorce niveles musterienses (Fumanal y Villaverde, 1988; Villaverde y Fumanal, 1990; Villaverde, 1992); únicamente contamos con una serie de dataciones por $\mathrm{C}^{14}$ sobre concreciones carbonatadas para el nivel $\mathrm{V}$ - final de Cova Negra $D$ - con un márgen amplio de indeterminación entre 29.000 y 34.000 BP (Villaverde y Fumanal, 1990). Los estudios sedimentológicos y polínicos han llevado a situar la última fase del yacimiento - Cova Negra F (niveles 1 a 3)- en los inicios del Würm III, estadio isotópico 2 (Villaverde, 1992; Villaverde et al. 1996), rectificando así las primeras impresiones que situaban el final de la secuencia a finales del Würm II (Villaverde, 1984) o no más allá del Würm II-III (Fumanal y Villaverde, 1988). De nuevo, nos encontramos con ausencia de dataciones cronométricas para los niveles directamente implicados en el problema.

Una atención especial merece el caso de Cova Beneito (Alicante) con una secuencia con cuatro niveles Musterienses (D4-D1), un nivel de discontinuidad $\mathrm{C}$, que contiene dos lentejones cenicientos adscribibles al Auriñaciense 0-C4-y al Auriñaciense Típico - C2 - (adscripción hipotética dada la escasez de material), dos niveles atribuibles al Auriñaciense evolucionado (B9 y B8), dos niveles Gravetienses, uno ProtoSolutrense, tres Solutrense Pleno y dos Solútreo-gravetienses secuencia tomada de Iturbe et al., 1993, que difiere tanto en número de niveles como en adscripciones culturales de las publicaciones anteriores (Iturbe y Cortell, 1982 y 1987). El yacimiento cuenta con cinco dataciones, cuatro de ellas provenientes de los niveles que nos interesan para estudiar el Final del Musteriense - C4 y D1- (Iturbe y Cortell, 1987; Iturbe y Cortell, 1992). EI Laboratorio de Tucson procesó por AMS muestras de carbón provenientes de $\mathrm{C} 4=33.900 \pm 1.100 \mathrm{BP}$ (AA-1388) y D1 = 38.800 \pm 1.900 (AA-1387); el Laboratorio de Gif-sur-Yvette procesó por $\mathrm{C}^{14}$ también carbones provenientes de los mismos niveles, obteniendo fechas para C4 $=26.040 \pm 890$ BP (Gif-7650) y para D1 $=30.160 \pm 680$ BP (GifTAN-89283), la diferencia entre los resultados de los dos laboratorios ronda los 8.000 años, sin embargo aunque los investigadores reconocen que las de Gif estarian reju- 
venecidas, ya que hubo problemas en la extracción de los ácidos húmicos (Iturbe y Cortell, 1992: 117; Carrión, Fumanal e Iturbe, 1993: 142; Iturbe et al., 1993: 82) aceptan estas fechas como correctas ya que encaja mejor con la interpretación sedimento-polínica y su adscripción a los inicios del Würm III convencional, estadio isotópico 2 . Otro problema añadido es la posible contaminación de las muestras por la existencia de carbono más jóven (Iturbe et al., 1993: 81). Además se han puesto en circulación dataciones $\mathrm{C}^{14}$ inéditas que dan fechas más antiguas para un nivel superior de $38.200+2.000-1.600 \mathrm{BP}$ (Gif-8307) y $37.930 \pm 330 \mathrm{BP}$ (Gif/LSM-8397) (Hublin et al. 1995).

Cova Beneito ofrece una serie de anomalías en su industria, alguna realmente importante, la industria lítica posee un alto índice de laminaridad y un mayor índice del Grupo del Paleolítico Superior o la presencia de dos fragmentos de lámina con retoque semiabrupto marginal, estos aspectos ya han sido documentados en otros sitios y no suelen plantear problemas ni generar dudas de su contexto, si exceptuamos la presencia de las láminas de dorso, además la industria ósea ha proporcionado un fragmento de diáfisis con marcas paralelas, un punzón bien trabajado, otra serie de huesos posiblemente trabajados aún en estudio y, sobre todo, un colgante sobre colmillo de lince perforado en la base de la raíz (Iturbe y Cortell, 1982; Iturbe y Cortell, 1992), hallazgo ciertamente problemático ya que es único dentro de los conjuntos Musterienses. Los investigadores plantean la presencia del colgante por préstamos de los Auriñacienses vecinos, al igual que el carácter evolutivo de la industria también se explica en términos de coexistencia (iturbe et al., 1993: 38 y 46). Esta hipótesis de posible aculturación es rechazada por la mayoría de los autores que no ven en los Musterienses tardíos de Levante y Andalucía ningún aspecto que les pueda acercar al Paleolítico Superior. Más bien, se ha propuesto para la presencia del colgante, una posible intrusión desde los niveles superiores (Villaverde, 1992: 62) o directamente consideran el nivel D1 como revuelto (d'Errico et al. 1998: 20).

Por último nos vamos a ocupar de los yacimientos portugueses, en primer lugar cabe destacar la Gruta Nova de Columbeira (Estremadura portuguesa), descubierta y excavada prácticamente en su totalidad en 1962 , en estas fechas se obtuvieron dos dataciones por $\mathrm{C}^{14}$ sobre "restos carbonosos" que hasta ahora habian permanecido inéditas (Zilhao, 1993: 134; Raposo y Cardoso, 1998: 57) al resultar demasiado jóvenes para las hipótesis del momento, teniendo en cuenta que por encima del nivel 7 hay otros cuatro niveles musterienses; el nivel $7 / 16$ dió $26.400 \pm 750$ BP (Gif2703) y el nivel $8 / 20=28.900 \pm 950 \mathrm{BP}$ (Gif-2704), estas fechas deberían tomarse con precaución dado el carácter atípico de la muestra utilizada 
para la datación. Cuatro nuevas fechas se han obtenido en el yacimiento sobre muestras dentales mediante $\mathrm{Th} / \mathrm{U}$, nivel $7=54.365-27.525+2.224$ (SMU-23851) y 35.876-35.583+27299 (SMU-235E1), nivel $8=101.487-$ $55919+38406$ (SMU-236E1) y 60.927-35.522+27405 (SMU-236E1), estas dataciones han sido rechazadas por el amplio márgen de error que arrojan (Raposo y Cardoso, 1998: 57).

Otras dataciones son ofrecidas para el nivel $\mathrm{K}$ de Caldeirao, 27.600 \pm 600 BP (OxA-1941), $18.060 \pm 140 \mathrm{BP}$ (OxA-5541) y $23.040 \pm 340 \mathrm{BP}$ (OxA-5521), estas fechas presentan un amplio márgen de oscilación además de resultar excesivamente recientes.

El yacimiento al aire libre de Foz do Enxarrique ha propocionao dataciones por Th/U sobre dientes de caballo, para su único horizonte paleolítico - con presencia de Elephas antiquus- de $32.938 \pm 1.055$ (SMU-225), $34.088 \pm 800$ (SMU-226) y $34.093 \pm 920$ (SMU-224). En cuanto a Figueira Brava, se obtuvo una datación por $\mathrm{C}^{14}$ sobre Patella sp. para el nivel 2 de $30.930 \pm 700$ BP (ICEN-387), aunque estas fechas fueron dadas como fiables por el laboratorio (Zilhao, 1993), debemos recordar que las determinaciones por radiocarbono basadas en conchas son bastante problemáticas.

A modo de resúmen, recordar 1 . que hay niveles directamente implicados en el problema que no cuentan con dataciones radiométricas (Carihuela, Cova Negra, Columbeira), 2. que otras fechas se obtuvieron con métodos aún experimentales (Carihuela), 3. que las muestras a veces no son apropiadas (Columbeira, Figueira Brava), 4. que hay dudas sobre la coherencia estratigráfica de algunos yacimientos (Beneito, Zafarraya) y 5. que no hay estudios sedimentoclimáticos completos para otros yacimientos (Bajondillo).

Debemos insistir en que para datar el período en cuestión, el $\mathrm{C}^{14}$ no parece el método más apropiado, ya que debido a su brevedad media, la capacidad práctica de los contadores de radiocarbono llega hasta los 35.000 años, por lo que debemos tomar estas fechas con precaución. Por otra parte, es evidente que se desechan sistemáticamente las fechas que resultan antiguas, y que permanecen inéditas las dataciones que no casan con la pervivencia del Musteriense en el estadio isotópico 2.

Sin querer entrar aquí en la discusión de las secuencias sedimentológicas y palinológicas, que serán objeto de un próximo trabajo, creemos que son necesarios estudios de comprobación de la diversidad paisajística y climática para realizar unas mejores correlaciones con las series clásicas del interpleniglaciar y los inicios de la degradación climática de la última glaciación. 
Si uno de los criterios básicos que se barajan para apoyar la hipótesis de una pervevencia del Musteriense y de sus autores, los Neandertales, en la mitad sur de la Península lbérica, son las dataciones radiométricas y estas en la mayoría de los casos son difíciles de sostener, quizá deberíamos comenzar a reexaminar los datos que poseemos.

\section{BIBLIOGRAFIA}

BarRoso, C. y HublíN, J.J. (1994): The Late Neanthertal site of Zafarraya (Andalucía, Spain). En F. Rodríguez et al. (eds.): Gibraltar during the Quaternary. AEQUA Monografías, 2. Sevilla: 61-70.

Carrión, J., Fumanal, M.P. y Iturbe, G. (1993): La secuencia polínica de Cova Beneito en su marco litoestratigráfico, arqueológico y geocronológico. En M.P. Fumanal y J. Bernabeu (eds.): Estudios sobre Cuaternario. Valencia: 139-148.

Cortés, M. y Simón, M.D. (1997): Cueva Bajondillo (Torremolinos, Málaga). Aportaciones al Paleolítico en Andalucía. En: J.M. Fullola y N. Soler (eds): El món mediterrari després del Pleniglacial (18.000-12.000 BP). ColAloqui. Banyoles 1995. Serie Monográfica, 17. Museu d'Arqueologia de Catalunya. Girona. págs. 275-219.

Cortés, M. et al. (1996): El Paleolítico en Andalucía. Córdoba.

DiAZ, F. (1994): Interferencias sedimentarias y cambios climáticos en Gorham's Cave (Gibraltar). En F. Rodríguez et al. (eds.): Gibraltar during the Quaternary. AEQUA Monografías, 2. Sevilla: 36-48.

D'ERRico, F. et al. (1998): Neanderthal acculturation in Western Europe? A critical review of the evidence and its interpretation. Current Anthropology. vol. 39. Supplement june: 1-44.

FREMLIN, J.H. (1974): Thermoluminiscence measurements of objects too old for carbon dating. Conference reports (Seminar at Boston): págs. 80-87.

Fumanal, M.P. y CaRRIón, J.S. (1992): El tánsito del Paleolítico Medio-Superior en la Cova de Beneito (Muro, Alicante). Avance del estudio estratigráfico y sedimentopolínico. En P. Utrilla (coord.): Aragón/Litoral Mediterráneo. Intercambios culturales durante la Prehistoria. Institución Fernando el Católico. Zaragoza: 107-116.

Fumanal, M.P. et Villaveroe, V. (1988): Cova Negra et le milieu du Paleolithique Moyen dans la region du Pays Valencien (Espagne). En M. Otte (ed.): L'Homme de Neandertal, vol. 2 L'environnement, Liège: 73-85.

Göksu, H.J. et al. (1974): Age determination of burned flint by a thermoluminiscent method. Science, 183: $651-654$.

Hoyos, M. et al. (1994): Sedimentación kárstica: procesos mortosedimentarios en la zona del Estrecho de Gibraltar. En F. Rodriguez et al. (eds.): Gibraltar during the Quaternary. AEQUA Monografías, 2. Sevilla: 36-48.

Hublin, J.J. et al. (1995): The Mousterian site of Zafarraya (Andalucia, Spain): dating and implicatios on the palaeolithic peopling processes of Western Europe. C.R. Académie des Sciences de Paris, t. 321, série lla: 931-937.

Iturbe, G. y Cortell, E. (1982): Cova Beneito: Avance preliminar. Saguntum, 17: 9-44.

ITURBE, G. y CORTELL, E. (1987): Las dataciones de Cova Beneito y su interés para el Paleolítico mediterráneo. Trabajos de Prehistoria, 44: 267-270.

ItURBe, G. y CoRTELL, E. (1992): El Musteriense Final Mediterráneo: nuevas aportaciones. En P. Utrilla (coord.): Aragón/Litoral Mediterráneo. Intercambios culturales durante la Prehistoria. Institución Fernando el Católico. Zaragoza: 117-127.

ITURBe, G. et al. (1993): Cueva Beneito (Muro, Alicante): una perspectiva interdisciplinar. Recerques del Museu D'Alcoi, II: 23-88.

MEDina, F. et al. (1986): Avance al estudio de los niveles musterienses de la cueva del Boquete de Zafarraya, Alcaucín, Málaga. (Excavaciones de 1981-83). En: Homenaje a Luís Siret. Consejería de Cultura. Junta de Andalucía: 94-105.

Raposo, L. y CARDoso, J.L. (1998): Las industrias líticas de la Gruta Nova de Columbeira (Bombarral, Portugal) en el contexto del Musteriense Final de la Península lbérica. Trabajos de Prehistoria, 55, n1 1: 39-62. 
VegA, L.G. (1988): El Paleolítico Medio del Sureste español y Andalucia Oriental. Col. Tesis Doctorales. Universidad Complutense de Madrid. 4 vols.

VEGA, L.G. (1990): La fin du Paléolithique Moyen au Sud de L'Espagne: ses implications dans le contexte de la Péninsule lbérique. En C. Farizy (ed.): Paléolitique moyen récent et paléolithique supérieur ancien en Europe. Mémoires du Musée de Préhistoire d'llle-de-France, 3: $169-179$.

VegA, L.G. (1993): El tránsito del Paleolítico Medio al Superior en el Sur de la Península Ibérica. En V. Cabrera (ed.): El origen del hombre moderno en el suroeste de Europa. U.N.E.D. Madrid: 147-170.

VEGA, L.G. y CARRION, J. (1993): Secuencia paleoclimática y respuesta vegetal durante el Pleistoceno Superior de la cueva de la Carihuela (Piñar, Granada, SE de España). En M.P. Fumanal y J. Bernabeu (eds.): Estudios sobre Cuaternario. Valencia: 131-138.

VEGA, L.G. et al. (1988): La séquence de la grotte de la Carihuela (Pínar, Granada): chronostratigraphie et paléoécologie du Pléistocène Supérieur au Sud de la Péninsule Ibérique. En M. Otte (ed.): L'Homme de Neandertal, vol. 2 L'environnement, Liège: 169-180.

VEGA, L.G. et al. (1997): Las industrias de la interfase Pleistoceno Medio-Superior en la cueva de la Carihuela (Pínar, Granada). En R. de Balbín y P. Bueno (eds.): II Cogreso de Arqueologia Peninsular. T.I. Paleolítico y Epipaleolítico. Fundación Rei Afonso Henriques: $105-118$.

Villaverde, V. (1984): La Cova Negra de Xátiva y el Musteriense de la Región Central del Mediterráneo Español. Servicio de Investigaciones Prehistóricas. Serie de Trabajos Varios, n1 79. Diputación Provincial de Valencia. Valencia.

VILlaVerde, V. (1992): El Paleolítico en el País Valenciano. En P. Utrilla (coord.): Aragón/Litoral Mediterráneo. Intercambios culturales durante la Prehistoria. Institución Fernando el Católico. Zaragoza: 55-87.

Villaverde, V. et Fumanal, M.P. (1990): Relations entre le Paléolithique Moyen et Le Paléolithique Supérieur dans le versant méditerranéen espagnol. En C. Farizy (ed.): Paléolitique moyen récent et paléolithique supérieur ancien en Europe. Mémoires du Musée de Préhistoire d'ille-de-France, 3: 177-183.

ZILHAO, J. (1993): Le passage du Paléolithique moyen au Paléolitique supérieur dans le Portugal. En V. Cabrera (ed.): El origen del hombre moderno en el suroeste de Europa. U.N.E.D. Madrid: $127-145$. 